EPJ Web of Conferences 32, 01010 (2012)

DOI: $10.1051 /$ epjconf/20123201010

(C) Owned by the authors, published by EDP Sciences, 2012

\title{
Dynamical modelling of neoclassical tearing mode suppression by ECCD
}

\author{
Bircan Ayten ${ }^{1, a}$ and Egbert Westerhof ${ }^{1}$ \\ FOM Institute DIFFER - Dutch Institute for Fundamental Energy Research, Association EURATOM- \\ FOM, Trilateral Euregio Cluster, PO Box 1207, 3430 BE Nieuwegein, The Netherlands
}

\begin{abstract}
The rotation of a magnetic island through the EC power deposition region in combination with a finite collisional time scale on which the EC power is converted into current are shown to affect the NTM stabilization by ECCD. In particular, the stabilizing term from localized ECCD in the generalized Rutherford equation oscillates at the island rotation frequency. As a consequence, also the island width oscillates at the rotation frequency. When the rotation frequency is reduced, these oscillations increase in amplitude and result in a net increase of the time averaged stabilizing effect of ECCD.
\end{abstract}

\section{Introduction}

The stabilization of neoclassical tearing modes (NTMs) by electron cyclotron current drive (ECCD) has been studied extensively in the framework of the generalized Rutherford equation (GRE) [1,2], which describes the evolution of the full width $w$ of the associated magnetic island. Including only the most relevant terms in the context of the present paper the GRE is written as [1]:

$$
0.82 \frac{\tau_{r}}{r_{s}} \frac{d w}{d t}=r_{s} \Delta_{0}^{\prime}(w)+r_{s} \Delta_{b s}^{\prime}+r_{s} \Delta_{C D}^{\prime}
$$

where $\tau_{r}=\mu_{0} r_{s}^{2} / \eta$ for the plasma resistivity $\eta$ at the resonant surface $r_{s}$ of the island. $\Delta_{0}^{\prime}(w)$ is the classical stability index. The driving mechanism of NTMs, which is the perturbation of the bootstrap current, is represented by the second term. The last term refers to the stabilizing effect of ECCD. Conventionally, $\triangle_{C D}^{\prime}$ is obtained by averaging the ECCD over an island rotation period $\tau_{\text {rot }}$. This requires that $\tau_{\text {rot }}$ is much shorter than the collisional time scale $\tau_{\text {coll }}$ on which the EC driven current is generated/decays. When this assumption is not valid, the EC driven current $\delta J_{C D}$ becomes time dependent, oscillating and moving through the island with the rotation period. As a result $\Delta_{C D}^{\prime}$ will oscillate with the rotation period as well.

Another relevant time scale is the characteristic time of island evolution, $\tau_{N T M}$. When the island evolution is slow, the time average of $\Delta_{C D}^{\prime}$ again yields the conventional expression. However, when the island evolves significantly during a rotation period, the oscillations of $\Delta_{C D}^{\prime}$ must be accounted for in the evolution of the island. In this paper we analyze the $\Delta_{C D}^{\prime}$ oscillations and their dependence on the different time scales, the island size and power deposition width. Furthermore, we study the consequences of the $\Delta_{C D}^{\prime}$ oscillations for the island evolution and for the minimum power requirement to fully suppress an island. When $\tau_{\text {coll }}$ and $\tau_{N T M}$ are normalized to $\tau_{\text {rot }}$, a two dimensional normalized parameter space results. In this parameter space we recognize two limits. On the one hand the regime of very fast rotation $\left(\tau_{N T M} / \tau_{\text {rot }} \gg 100\right)$ in which $\Delta_{C D}^{\prime}$ may be obtained through an average over a rotation period [3-6]. On the other hand, the locked mode regime $\left(\tau_{N T M} / \tau_{r o t} \ll 1\right)$ in which the island does not rotate. This work focuses on the rest of the parameter space.

\footnotetext{
a e-mail: B.Ayten@differ.nl
} 


\section{Generalized Rutherford equation}

We will be presenting the results in terms of a normalized GRE. Time is normalized to $\tau_{N T M}$ which is identified with the growth rate coming from the maximum of the bootstrap term, i.e.

$$
\tau_{N T M} \equiv \frac{0.82 \tau_{r}}{r_{s} \Delta_{b s, \max }^{\prime}} .
$$

Spatial scales like island sizes and deposition widths are normalized by $r_{s}$. The classical stability index $\Delta_{0}^{\prime}$ is expressed in terms of the saturated island size, $w_{\text {sat }}$ at which the NTM growth saturates in the absence of other stabilizing effects, i.e.

$$
\Delta_{0}^{\prime}=-\Delta_{b s}^{\prime}\left(w=w_{s a t}\right) .
$$

Finally, the small island limit of the bootstrap term is supposed to be determined by the effects of incomplete pressure flattening from the competition between parallel and perpendicular transport [7]. This way the GRE is obtained in its following normalized form,

$$
\frac{d \bar{w}}{d \bar{t}}=-\frac{2 \bar{w}_{\text {sat }} \bar{w}_{\text {marg }}}{\bar{w}_{\text {sat }}^{2}+\bar{w}_{\text {marg }}^{2}}+\frac{2 \bar{w} \bar{w}_{\text {marg }}}{\bar{w}^{2}+\bar{w}_{\text {marg }}^{2}}+\bar{\Delta}_{C D}^{\prime},
$$

where $\bar{t} \equiv t / \tau_{N T M}, \bar{w} \equiv w / r_{s}$ and $\bar{\Delta}_{C D}^{\prime}=\Delta_{C D}^{\prime} / \Delta_{b s, \max }^{\prime}$. The contribution of ECCD is written as [4-6]

$$
r_{s} \Delta_{C D}^{\prime}=-\frac{16 \mu_{0} L_{q} r_{s}}{B_{p} \pi \omega^{2}}\left[\int_{-\infty}^{\infty} d x \oint d \xi J_{C D} \cos \xi\right] .
$$

To evaluate this term as a function of time a proper equation for the evolution of the driven current density is needed taking into account the effects of rotation and a finite collision time.

This dynamical modelling of the electron cyclotron current drive is performed as follows. The power deposition profile is written as the product of the total power $P_{t o t}$ with a normalized profile function, $\tilde{p}_{C W}(x, \xi)$ defined as

$$
\tilde{p}_{C W}(x, \xi)=\frac{1}{\Delta \xi \pi^{3 / 2} w_{d e p} R r_{s}} e^{-4\left(r-r_{d e p}\right)^{2} / w_{d e p}^{2}} \Pi(\xi),
$$

with a Gaussian radial profile of full width $w_{d e p}$ centered at $r_{d e p}$ and a helical profile defined by the top hat function $\Pi(\xi)$,

$$
\begin{gathered}
\Pi(\xi)=1, \omega_{\text {rot }} t-\Delta \xi / 2 \leq \xi \leq \omega_{\text {rot }} t+\Delta \xi / 2, \text { modulus } 2 \pi \\
\Pi(\xi)=0, \text { otherwise, }
\end{gathered}
$$

which at $t=0$ is centered at $\xi=0$ with a helical width $\Delta \xi$. Here, $\omega_{\text {rot }}=2 \pi / \tau_{\text {rot }}$ is the angular rotation frequency of the island. Note, that in this work we will only consider CW application of the ECCD power, with a radial deposition that is perfectly aligned with the resonant radius of the NTM, i.e. $r_{d e p}=r_{s}$.

At this point it is convenient to define the symmetric island topology adopted for this work: $\Omega=$ $8 x^{2} / w^{2}-\cos \xi$ is the normalized flux surface label with $\Omega=-1$ at the O-point of the island and $\Omega=1$ at the X-point. Here, $x=r-r_{s}$ is the displacement from the resonant surface. Outside the island the label $\sigma=\operatorname{sgn}(x)$ distinguishes the two different surfaces with identical $\Omega$ on opposite sides of $r_{s}$.

Parallel transport is assumed to be virtually instantaneous, such that the driven current density is a flux function. The ECCD efficiency is assumed to be a simple constant, $\eta_{C D} \equiv I_{C D} / P_{t o t}$. The driven current density then depends only on the flux surface averaged power density $P_{E C}(\Omega, t)$ as a function of time. Because the island evolution is incompressible, the evolution of the island will not affect the current density $J_{C D}\left(S_{\Omega}\right)$ expressed as a function of the total area enclosed by the flux surface: $J_{C D}\left(S_{\Omega}\right)$ 
decays on a collisional time scale $\tau_{\text {coll }}$ and is generated by the instantaneous power deposited on that surface, i.e.

$$
\frac{\partial J_{C D}\left(S_{\Omega}, \sigma, t\right)}{\partial t}=-\frac{J_{C D}\left(S_{\Omega}, \sigma, t\right)}{\tau_{\text {coll }}}+2 \pi R \frac{\eta_{C D}}{\tau_{\text {coll }}} P_{E C}\left(S_{\Omega}, \sigma, t\right) .
$$

Only on those surfaces that are being reconnected due to island growth the current density must be averaged over the two contributing surfaces, while on surfaces that get disconnected due to island shrinkage the current density is conserved:

For $S_{\text {sep }}\left(t^{-}\right)<S_{\Omega} \leq S_{\text {sep }}\left(t^{+}\right)$:

$$
J_{C D}\left(S_{\Omega}, t^{+}\right)=\frac{1}{2}\left(J_{C D}\left(S_{\Omega}, \sigma=1, t^{-}\right)+J_{C D}\left(S_{\Omega}, \sigma=-1, t^{-}\right)\right),
$$

where $S_{\text {sep }}$ represents the enclosed surface area within the separatrix and $t^{-}$and $t^{+}$refer to the times just before and just after the surfaces are reconnected, respectively.

For $S_{\text {sep }}\left(t^{+}\right)<S_{\Omega} \leq S_{\text {sep }}\left(t^{-}\right)$:

$$
J_{C D}\left(S_{\Omega}, \sigma= \pm 1, t^{+}\right)=J_{C D}\left(S_{\Omega}, t^{-}\right),
$$

where $t^{-}$and $t^{+}$now refer to the times just before and just after the surfaces are disconnected. Equations Eq. (9), Eq. (10) and Eq. (11) describe the time dependent evolution of the driven current in the case of an evolving island.

\section{$3 \triangle_{C D}^{\prime}$ evolution at constant island width}

In this section we solve equations Eq. (9) and Eq. (5) for constant island width $w$. The results of the $\Delta_{C D}^{\prime}$ calculations are normalized to a reference $\Delta_{R E F}^{\prime}$, which is obtained in the case where the power is evenly distributed over all phases of the island and $\tau_{\text {coll }}=0$ :

$$
\Delta_{R E F}^{\prime} \equiv \Delta_{C D}^{\prime}\left(\Delta \xi=2 \pi, \tau_{\text {coll }}=0\right) .
$$

This reference case corresponds to $\Delta_{C D}^{\prime}$ as obtained conventionally by averaging the power deposition and current drive profiles over a rotation period [4-6]. When $\tau_{\text {coll }}$ is different from zero, the EC driven current takes time to reach a quasi-steady state [8]. Consequently the same holds for $\Delta_{C D}^{\prime}$ as shown in Fig. 1(a) for a fast rotating island with $\tau_{\text {coll }}=3 \tau_{\text {rot }}$ and $w=w_{\text {dep }}$. The solid curve represents the case for which the power is spread evenly over all phases of the island. For $t>>\tau_{\text {coll }}$ a steady state is reached identical to $\Delta_{R E F}^{\prime}$. Results for $\Delta_{C D}^{\prime}$ calculations with a small helical power deposition width of $(0.02 \times 2 \pi)$ are depicted by the dashed curve. The helically localized power deposition results in oscillations of the driven current density profile and of $\Delta_{C D}^{\prime}$ at the plasma rotation frequency. For $t>>\tau_{\text {coll }}$ a quasi-steady state is reached with periodic oscillations of $\Delta_{C D}^{\prime}$ around its reference value. The $\Delta_{C D}^{\prime}$ oscillations in the quasi-steady state are shown in Fig. $1(\mathrm{~b})$ for $\tau_{\text {coll }} / \tau_{\text {rot }}=0,0.3,1.0$, and $\infty$. The case of $\tau_{\text {coll }} / \tau_{\text {rot }}=0$ can also be seen as representing the $\Delta_{C D}^{\prime}$ efficiency as a function of island phase in case of a locked mode. As $\tau_{\text {coll }} / \tau_{\text {rot }}$ increases, the amplitude of the oscillations decreases. In addition, a phase shift of the $\Delta_{C D}^{\prime}$ oscillation relative to the island rotation is created. As equations Eq. (9) and Eq. (5) are linear, the time average over a full rotation period of the CD term, $\left\langle\Delta_{C D}^{\prime}\right\rangle_{\tau_{\text {rot }} \text {, }}$, in the quasi steady state is identical to the reference case: $\lim _{t \rightarrow \infty}\left\langle\Delta_{C D}^{\prime}\right\rangle_{\tau_{\text {rot }}}=\Delta_{R E F}^{\prime}$. This is born out by the numerical calculations up to numerical accuracy.

The extrema of $\Delta_{C D}^{\prime}$ simulations at quasi-steady state as a function of $w / w_{\text {dep }}$ for three different $\tau_{\text {coll }} / \tau_{\text {rot }}$ are shown in Fig. 2(a). The results are shown in terms of extrema and not simply the oscillation amplitudes, because the periodic $\Delta_{C D}^{\prime}$ oscillation is not a simple sinusoidal oscillation around its reference value. Figure 2(b) gives the maximum and the minimum as a function of $\tau_{\text {coll }} / \tau_{\text {rot }}$ for the fixed island width $\left(w=w_{d e p}\right)$. For $\tau_{c o l l} / \tau_{\text {rot }}<10^{-1}$ the extrema in $\Delta_{C D}^{\prime}$ are seen to saturate at a maximum value. As $\tau_{\text {coll }} / \tau_{\text {rot }}$ is increased above $10^{-1}$, the extrema quickly approach the reference value, leaving only modest oscillations for $\tau_{\text {coll }} / \tau_{\text {rot }}>1$. The amplitude of the $\Delta_{C D}^{\prime}$ oscillations remain almost unchanged when the helical power deposition width is increased up to $\Delta \xi=0.2 \times 2 \pi$. 

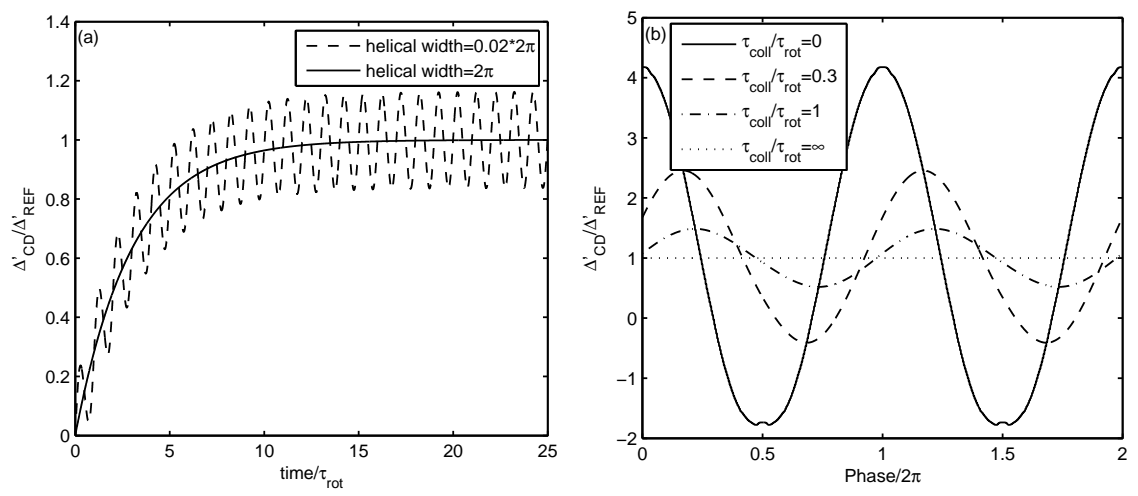

Fig. 1. (a) $\Delta_{C D}^{\prime}$ calculations for $\tau_{\text {coll }}=3 \tau_{\text {rot }}$ and $w=w_{\text {dep }}$ as a function of time till a quasi-steady state is reached. The result of the case where the power is distributed evenly over all phases of the island is given by the solid curve. The dashed curve represents $\Delta_{C D}^{\prime}$ for a power deposition helical width $2 \%$ of the whole island helical width. (b) $\Delta_{C D}^{\prime}$ oscillations in quasi-steady state for $\Delta \xi=0.02 \times 2 \pi$ and $w=w_{\text {dep }}$ for different values of $\tau_{\text {coll }} / \tau_{\text {rot }}=0,0.3$, 1.0 , and $\infty$.
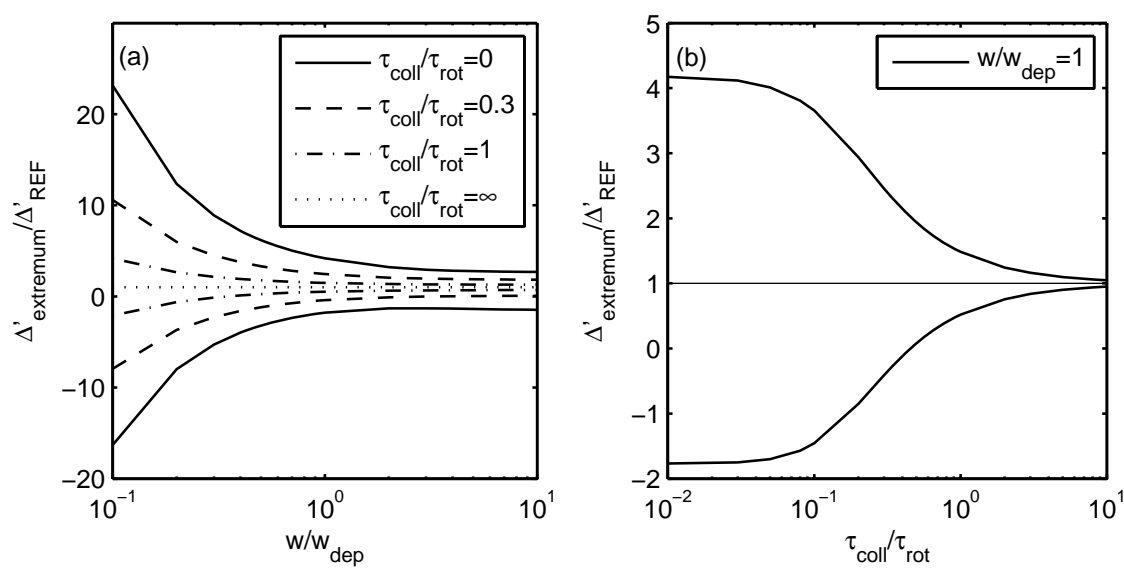

Fig. 2. (a)The dependence of extrema of $\Delta_{C D}^{\prime}$ on $w / w_{\text {dep }}$ is calculated for different $\tau_{\text {coll }} / \tau_{\text {rot }}$ values. The dotted line for which the $\tau_{\text {coll }} / \tau_{\text {rot }}=\infty$ is identical to the reference case. (b)Extrema of $\Delta_{C D}^{\prime}$ for a fixed island width $\left(w=w_{\text {dep }}\right)$ as a function of $\tau_{\text {coll }} / \tau_{\text {rot }}$.

\section{Dynamical modelling with self consistent island width evolution}

We now investigate how the oscillations in $\Delta_{C D}^{\prime}$ influence the stabilization of NTMs. The GRE is used in its normalized form Eq. (4). A number of cases are selected in the 2D parameter space spanned by $\tau_{\text {coll }} / \tau_{\text {rot }}$ and $\tau_{N T M} / \tau_{\text {rot }}$. The normalized saturated island, marginal island, and EC deposition width are chosen as representative of a 2/1 NTM in ITER: $\bar{w}_{\text {sat }}=0.2$ and $\bar{w}_{\text {marg }}=0.0125$, with a narrow EC power deposition profile $\bar{w}_{d e p}=0.01$ and helical width $\Delta \xi=0.02 \times 2 \pi$. The reference case $\left(\Delta \xi=2 \pi, \tau_{\text {coll }}=0\right)$ is used to derive a minimum required power $P_{\min }$ for NTM stabilization. In Fig. 3 we show the results of the simulations with an ECCD power marginally above $P_{\min }$ for $\tau_{\text {coll }} / \tau_{\text {rot }}=0$ and various values of $\tau_{N T M} / \tau_{r o t}=1,10$ and 100. The effect of rotation is an oscillation in the island width, whose amplitude increases proportional to the rotation period. This oscillation is seen to result in a net increase of the stabilizing effect of ECCD and a consequent reduction in the time required for full suppression of the mode. 


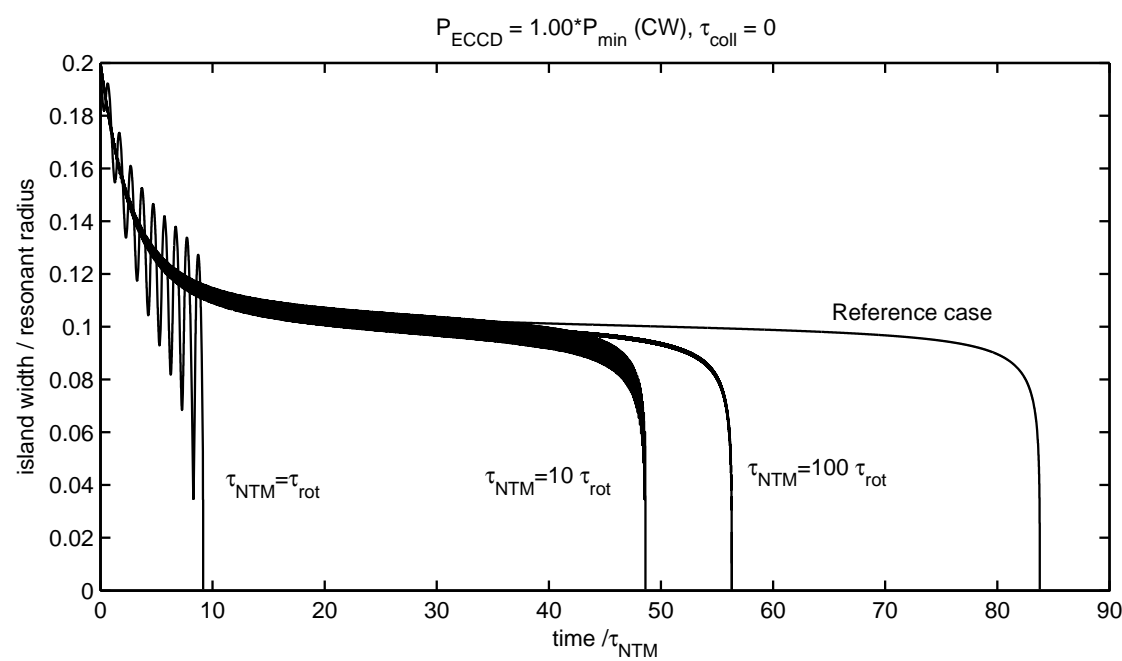

Fig. 3. The island width evolution simulations with a power marginally above $P_{\min }(C W)$ in the cases of reference, $\tau_{N T M} / \tau_{\text {rot }}=1,10$ and 100 , for each of them $\tau_{\text {coll }} / \tau_{\text {rot }}=0$. Other parameters are given in the text.

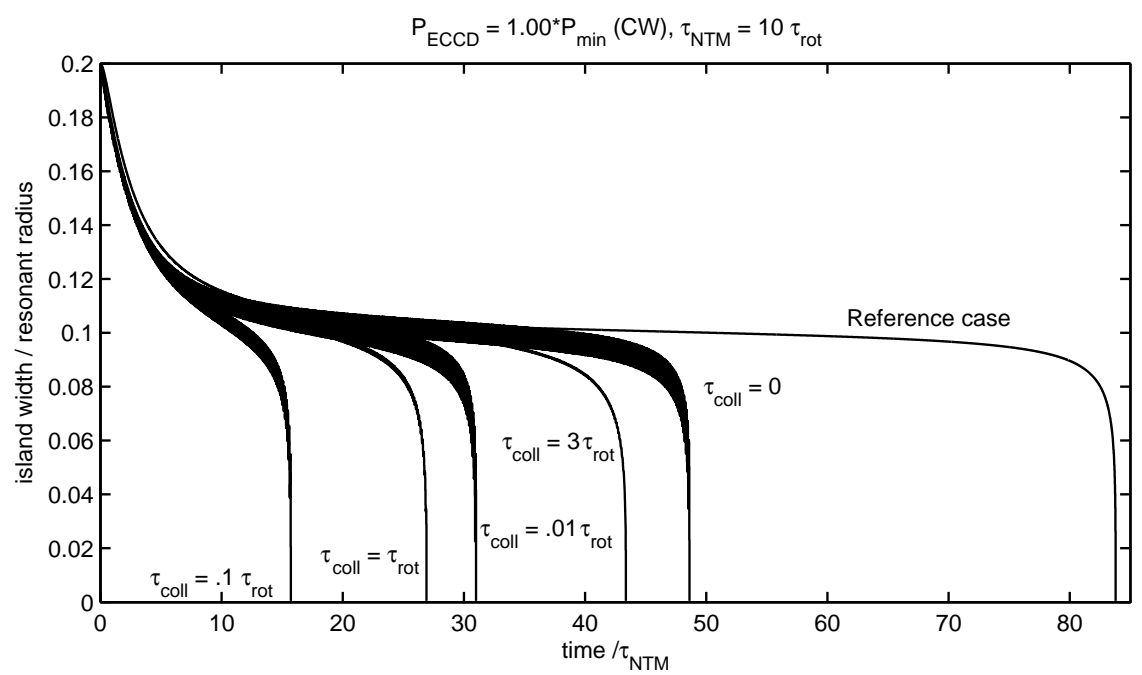

Fig. 4. The island width evolution simulation with a power marginally above $P_{\min }(C W)$ for $\tau_{N T M} / \tau_{\text {rot }}=10$ and different $\tau_{\text {coll }} / \tau_{\text {rot }}$ values. Additionally, the evolution in the reference case is included.

In a second set of simulations we studied the effect of introducing a finite collision time. To this end, we performed simulations with the same parameters as in case of Fig. 3 and $\tau_{N T M} / \tau_{\text {rot }}=10$ but now for various values of $\tau_{\text {coll }} / \tau_{\text {rot }}=0,0.01,0.1,1$, and 3 . As shown by the results given in Fig. 4 , the finite collision time at first results in a further stabilizing effect of the ECCD. The largest stabilizing effect is obtained for $\tau_{c o l l} / \tau_{r o t}=0.1$ As the collision time is increased, the oscillations in $\Delta_{C D}^{\prime}$ and consequently in the island width are reduced. Also the minimum power required for full suppression is reduced below the value of $P_{\min }$ in the reference case. In case of $\tau_{N T M} / \tau_{\text {rot }}=1$ and $\tau_{\text {coll }} / \tau_{\text {rot }}=0$, the minimum required power for full NTM stabilization is reduced to $95 \%$ of $P_{\min }$. This effect is larger when the EC power deposition is wider. 


\section{EPJ Web of Conferences}

\section{Conclusion}

In this paper the oscillations in $\Delta_{C D}^{\prime}$ originating from the rotation of the island though the EC power deposition region and their dependence on $\tau_{c o l l}, \tau_{\text {rot }}$, the island size and the power deposition width have been investigated. Furthermore, their consequences for the island growth or the stabilization are analyzed in the 2D normalized parameter space spanned by $\tau_{\text {coll }} / \tau_{\text {rot }}$ and $\tau_{N T M} / \tau_{\text {rot }}$. This work shows that the net result of the oscillations in $\Delta_{C D}^{\prime}$ is a slight increase in the stabilizing effect of ECCD and consequently, a reduction in the minimum power requirement to fully suppress an island.

The $\Delta_{C D}^{\prime}$ calculations show that as $\Delta_{C D}^{\prime}$ is proportional to $\delta J_{C D}$, it evolves on the time scale of $\tau_{c o l l}$. It takes time for $\Delta_{C D}^{\prime}$ to reach a quasi-steady state depending on the value of $\tau_{\text {coll }}$. The helically localized power deposition on a rotating magnetic island leads to oscillations both in the driven current density and in $\Delta_{C D}^{\prime}$ at the plasma rotation frequency. Once the quasi-steady state is reached, $\Delta_{C D}^{\prime}$ oscillates around its reference value. Because the current drive is assumed to be in the linear regime, the time average over a full rotation period of $\Delta_{C D}^{\prime}$ in the case of a constant island width is exactly equal to its reference value regardless of $\tau_{\text {coll }} / \tau_{\text {rot }}$. The amplitude of the oscillations increases both with decreasing $\tau_{\text {coll }} / \tau_{\text {rot }}$ for a fixed island width as well as with decreasing $w / w_{\text {dep }}$ for a given $\tau_{\text {coll }} / \tau_{\text {rot }}$. The oscillations in $\Delta_{C D}^{\prime}$ are damped for $\tau_{\text {coll }} / \tau_{\text {rot }} \gg 10$. Furthermore, as $\tau_{\text {coll }} / \tau_{\text {rot }}$ increases, the phase shift of the $\Delta_{C D}^{\prime}$ oscillation relative to the island rotation increases. The case of $\tau_{\text {coll }} / \tau_{\text {rot }}=0$ also represents the efficiency of $\Delta_{C D}^{\prime}$ as a function of island phase for a locked mode. The oscillations in $\Delta_{C D}^{\prime}$ are affected by $\Delta \xi$ as well. The amplitude of these oscillations increases as the helical width decreases saturating for helical widths of $0.2 \times 2 \pi$ or smaller.

The dynamical modelling with self consistent island width evolution shows that the effect of rotation is an oscillation in the island width with the rotation period. A decrease in the NTM rotation frequency increases the amplitude of this oscillation. The oscillation in $\Delta_{C D}^{\prime}$ intensifies the net stabilizing effect of the ECCD decreasing the time required for full suppression of the mode. Introducing a finite collision time at first provides an extra stabilizing effect. Further increase in the collision time lowers the oscillations in $\Delta_{C D}^{\prime}$ and also in the island width. Also the minimum power required for full suppression is reduced below the value of $P_{\min }$ in the reference case. The reduction is larger for the case of wide ECCD deposition.

\section{References}

1. R.J. La Haye. Physics of Plasmas, 13(5), May 2006.

2. R. Prater. Physics of Plasmas, 11(5):2349, 2004.

3. F.W. Perkins, R.W. Harwey, M. Mahowski, and M.N. Rosenbluth. In Proc. $24^{\text {st }}$ EPS Conference on Plasma Physics, Berchtesgaden, 9 - 13 June 1997, pages P-III. 1017. ECA vol. 21A, 1997.

4. C.C. Hegna and J.D. Callen. Physics of Plasmas, 4(8):2940-2946, August 1997.

5. O. Sauter. Physics of Plasmas, 11(10):4808-4813, 2004.

6. D. De Lazzari and E. Westerhof. Nuclear Fusion, 49(7):075002, July 2009.

7. R. Fitzpatrick. Physics of Plasmas, 2(3):825-838, March 1995.

8. G. Giruzzi, M. Zabiégo, T.A. Gianakon, X. Garbet, A. Cardinali, and S. Bernabei. Nuclear Fusion, 39(1):107, 1999.

Acknowledgement The work in this paper has been performed in the framework of the NWO-RFBR Centre of Excellence (grant 047.018.002) on Fusion Physics and Technology. This work, supported by the European Communities under the contract of Association between EURATOM/FOM, was carried out within the framework of the European Fusion Programme. The views and opinions expressed herein do not necessarily reflect those of the European Commission. 